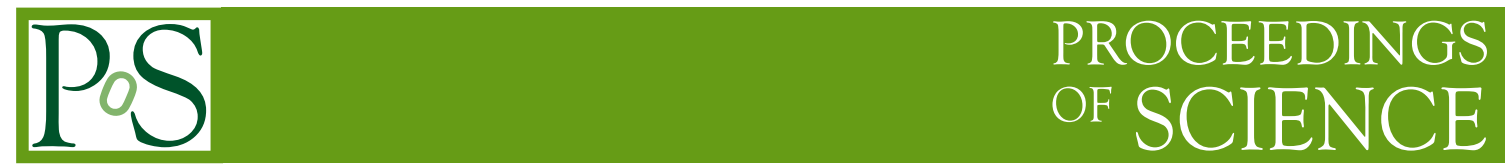

\title{
Rho Meson in Unquenched Lattice QCD
}

\author{
Mushtaq Loan* \\ International School, Jinan University, Guangzhou 510632, China \\ E-mail: mushe@ jnu.edu.cn
}

\begin{abstract}
We study the meson correlators with two degenerate flavours of Wilson fermions at finite temperature on improved anisotropic lattice. We analyse the lowest peak mass and width of pseudo-scalar and vector mesons around the transition phase. Our preliminary results suggest signals of melting resonance contribution of meson spectral functions in the deconfined phase.
\end{abstract}

The XXVIII International Symposium on Lattice Field Theory, Lattice2010

June 14-19, 2010

Villasimius, Italy

${ }^{*}$ Speaker. 


\section{Introduction}

The study of hadron properties at finite temperature plays an important role for the understanding of the QCD phase transition. With increasing temperature it is expected that hadrons change their properties around QCD phase transition [1]. The thermal modification of masses and decay widths of hadrons is one of the central issues in the discussion of experimental signals that can emerge in high energy heavy ion collision experiments [2]. The vector mesons deserve special attention as they couple directly to virtual photons, which can decay into dileptons. This process could be helpful in carrying the information about possible-in-medium changes of vector mesons to the detectors. Numerical simulations based on the lattice QCD can, in principle, provide this information through the analysis of thermal correlators of suitably chosen operators carrying the hadronic quantum numbers.

Accurate resolution of static observables, like hadron masses and decay constants at zero temperature, through recent lattice calculations with satisfactory precision, has been established quite well $[3,4,5]$. At finite temperature the access to dynamical quantities, in particular real-time correlation function is highly desirable but this has proven difficult. Previous quenched lattice simulations $[6,7,8,9,10]$ with staggered as well as Wilson fermions gave a somewhat fixed picture of the evidence for significant changes of meson masses around the transition phase. This is likely due to the quenched approximation and large quark masses used in these studies. In order to study the observed modifications of hadron correlators in presence of a thermal medium, a detailed study of the thermal properties of hadrons is required. In the present work we advance an attempt towards simulation of full QCD to explore the possibility if the observed modifications of meson correlators are indeed related to the disappearance of meson bound states.

\section{Numerical Details}

To avoid the large amount of computation necessary in full QCD, we use the improved lattice actions, which are designed to reduce scaling violation and hence should allow meaningful results from course lattice spacing. We choose to use the improved anisotropic tadpole-improved Syamnzik gauge action [11] and clover fermion action [12] for full QCD configurations. We generate the dynamical configurations for two flavours of degenerate light quarks with the Hybrid Monte Carlo algorithm on $12^{3} \times N_{t}\left(N_{t}=16,20,24,28\right)$ lattices for the couplings $\beta=2.0$ and 2.05. For the inversion of the quark matrix during HMC update we use even-odd preconditioned BICGSTAB algorithm. The bare gauge and fermion anisotropies are tuned to satisfy the renormalized anisotropy $\xi=2$ and hopping parameters are chosen in the range $0.1445-0.1452$. The Polyakov loops are measured at each trajectory and meson propagators are measured at each 5 trajectories.

Considering the success of smearing for an optimized projection onto the ground state at zero temperature, one might be tempted to use this method also at finite temperature where peaks are less pronounced compared to the continuum contributions. For $T>T_{c}$ it is very uncertain to project on an actual ground state, since even the smearing of free meson correlator function leads to sharp peaks instead of the broad continuum. The smearing causes the reduced curvature of the correlator at small $t$ separations, which translates into unphysical peaks in the spectral function. Therefore, 
to preserve the actual information about the ground state, we use the point-point correlator at finite temperature.

We determine the critical temperature $T_{c}$ by measuring the Polyakov loop susceptibility $\chi_{L}$ at $N_{t}=22,24$ and 26 at $\beta=2.05$. The loop susceptibility was also measured for various values of hopping parameter at fixed $N_{t}=24$ and $\beta=2.05$. The peak position of $\chi_{L}$ is obtained at about $N_{t}=24$ and $\beta=2.05$. To express the crossover temperature in physical units we used rho mass at zero temperature. The preliminary result for the critical temperature is obtained as $T_{c} \simeq 184(5)$ $\mathrm{MeV}$. Our value for $T_{c}$ is in quantitative agreement with the results obtained in [13, 14]. The pseudo-scalar and vector meson correlators at finite temperature are measured at three values of temporal lattice extent, $N_{t}=28,20$ and 16 . The corresponding temperatures are $0.88 T_{c}, 1.55 T_{c}$ and $2.03 T_{c}$, respectively.

\section{Finite Temperature Analysis}

The mass and the width of the lowest spectral component are extracted by using $\chi^{2}$ fit method with Breit-Wigner (BW)+pole form. This form ensures the suppression of contribution from high frequencies. The statistical errors are estimated by jackknife analysis. The fit is applied to the point correlators in the fit range $\left[t_{\min }, t_{\max }\right]$ where the results show negligible $t$ dependence, i.e., contribution of the ground state dominates. We choose the fit range where effective mass and width show simultaneous plateaus. The results for effective mass and width are given in Table 1. We quote our results in lattice units to avoid the uncertainty in lattice cutoff.

Table 1: The best-fit mass $m_{e f f}$ and width $\Gamma$ of pseudo-scalar and vector meson channels through BW+pole ansatz at various temperatures.

\begin{tabular}{ccccc}
\hline \hline \multicolumn{2}{c}{$\mathrm{V}$} & \multicolumn{2}{c}{ PS } \\
\hline$T / T_{c}$ & $m_{e f f}$ & $\Gamma$ & $m_{e f f}$ & $\Gamma$ \\
\hline 0.88 & $0.188(3)$ & $0.0032(3)$ & $0.179(2)$ & $0.0032(1)$ \\
1.55 & $0.184(4)$ & $0.0037(4)$ & $0.182(3)$ & $0.0033(3)$ \\
2.02 & $0.183(6)$ & $0.0044(5)$ & $0.183(3)$ & $0.0043(4)$ \\
\hline
\end{tabular}

\subsection{Meson correlators at $T=0.88 T_{c}$}

Analysing the thermal correlators at finite temperature, we notice that both the pseudo-scalar and vector correlators show a clear exponential decay with time, thereby signalling a sharp peak in the spectral functions. The results of the effective mass and width of the ground state peak are shown in Fig. 1. In both cases the fit ranges start at earlier times and last for quite few a time slices. Observing the dependence of mass and width parameters on the lower bound of the fit range, we fix $t$ range to $t_{\min }=4$ and $t_{\max }=10$. It is obvious from the figure that $\mathrm{BW}+$ pole form exhibits stable results both for the pseudo-scalar and vector channels for $t_{\min } \geq 4$. The width of the vector meson seems to remain stable with increasing $t_{\min }$, which indicates the finite width for the spectral function at this temperature. On the other hand, the width of the PS channel decrease at large $t$ separation indicating a strong peak with vanishing width (Fig. 1b). This is further explored in 
terms of the spectral function by using the standard $\chi^{2}$ fit with BW+pole ansatz for shape of the spectral function in Fig.1c. It is evident that the pseudo-scalar spectral function shows rather sharp ground state peak whereas the vector spectral function shows a slightly broader peak with reduced height which is in accordance with the analysis of effective width of vector meson.
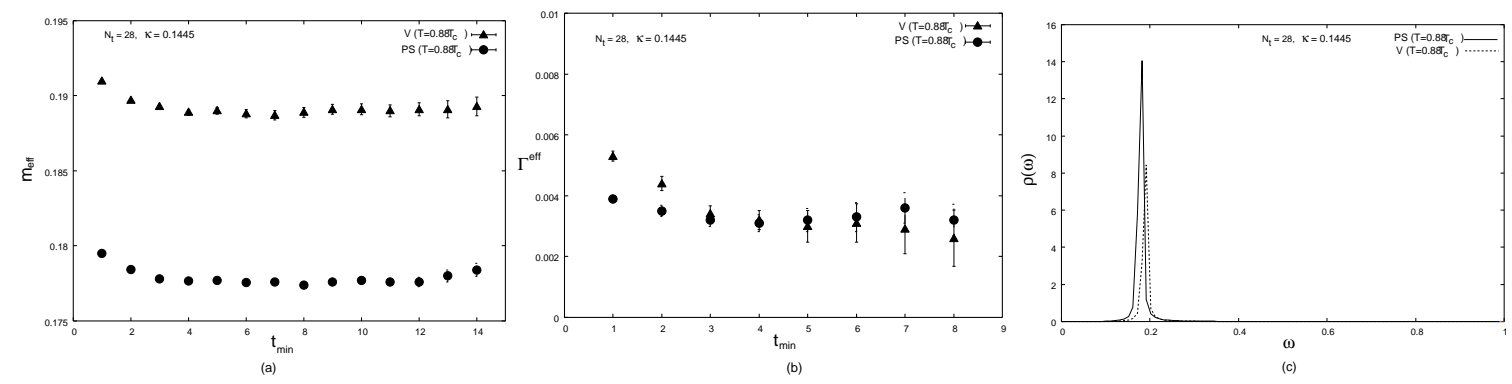

Figure 1: Results of BW+pole fit for the (a) ground state mass (b) lowest peak width (c) spectral shape in PS and V channels at $T=0.88 T_{c}$ and $\kappa=0.1445$.

It is not clear whether this thermal change in vector correlator is an in-medium induced modification of the behaviour of the correlator or only a lattice artifact due to limited statistics or relatively large quark masses used near critical temperature from below.

3.2 Thermal mesons at $T=1.55 T_{c}$ and $T=2.03 T_{c}$

Using a point-point correlator for $T>T_{c}$ for the reason described in Sec. I, we compare our results for effective widths at $T=1.55 T_{c}$ with the corresponding results at $T=0$ in Fig. 2a). We notice that the peak width of the vector meson is significantly increased. This slight temperature effect is seen to continue in mass and width parameters at $T=2.03 T_{c}$ (Figs. $2 \mathrm{~b}$ and $2 \mathrm{c}$ ). In both cases, the fit range starts at earlier times and the observables do not show a serious $t$ dependence, which indicates that the meson operators couple exclusively to the lowest spectral components. We observe that statistical fluctuation of the peak mass in the pseudo-scalar channel is much smaller than that for vector meson. The effect is visible in the large error bars on the vector masses. The results indicate that the widths associated with the ground state peaks are finite and mass shifts are significant in both channels.
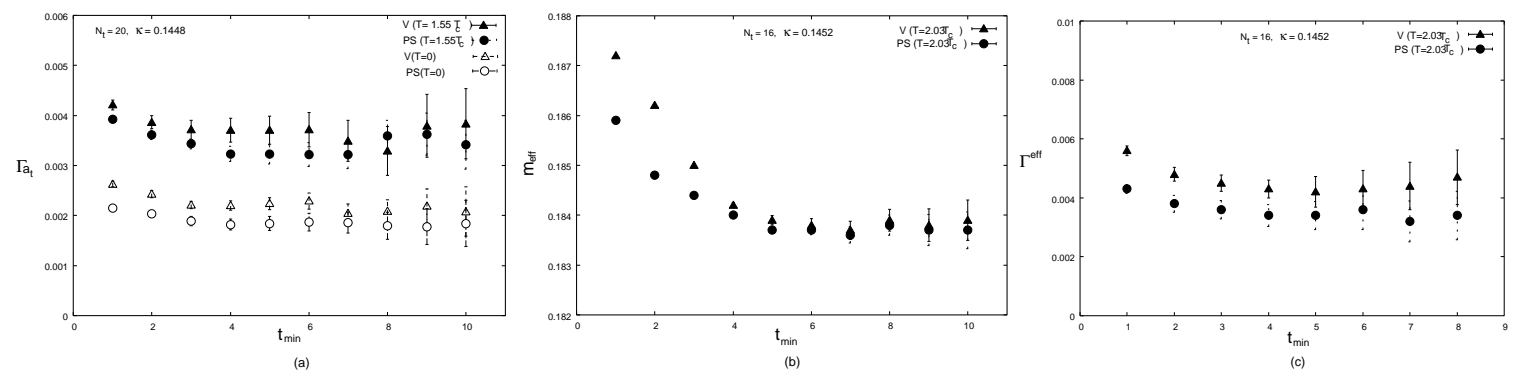

Figure 2: Results at $T>T_{c}$ for the PS and V channels, (a) comparison of effective widths at $T=1.55 T_{c}$ with the corresponding results at $T=0$ (b) lowest peak mass at $T=2.03 T_{c}$ and (c) lowest peak width at $T=2.03 T_{c}$.

Based on BW+pole ansatz, the best fit $\Gamma$ of the spectral function are shown as function of temperature in Fig. 3a. It is clear from the figure that while the temperature dependence of width 
is only mild below $T_{c}$, the thermal widths show a significant change as temperature increases. The vector meson ground state broadens considerably and shifts towards higher energies above the critical temperature, indicating a genuine in-medium modifications of the thermal correlators in the deconfined phase.

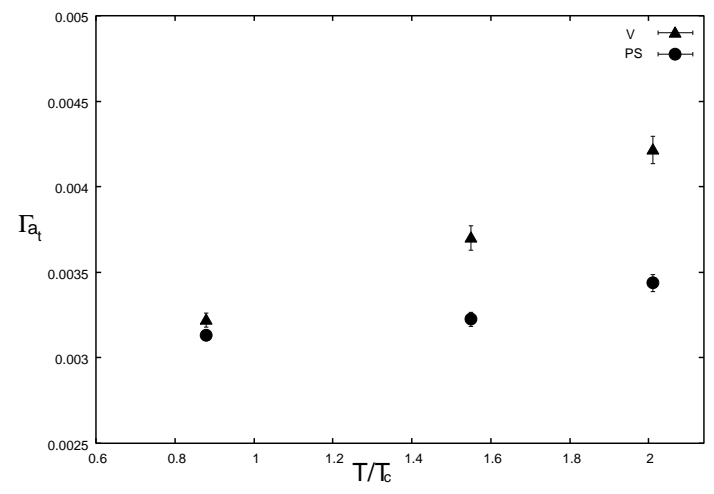

(a)

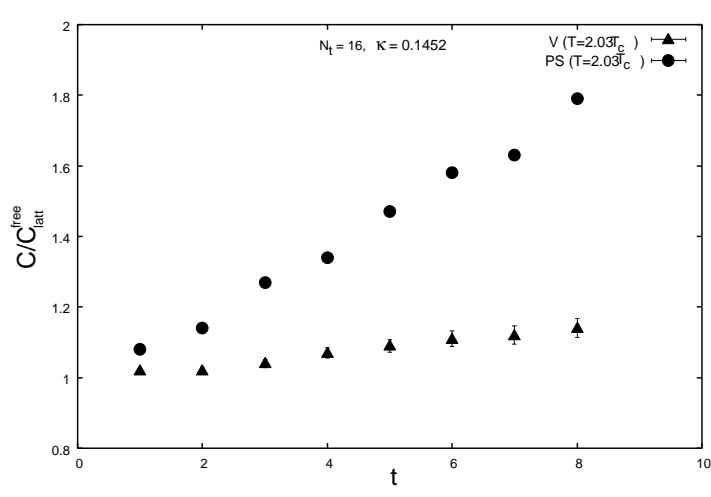

(b)

Figure 3: (a) Temperature dependence of the width (b) ratio of meson and free thermal meson correlators on the lattice.

At high temperature a scaling of the spectral function with temperature is expected and one would expect that the quark propagators should be close to the free ones. Therefore it is physically more motivating to look for the unbound quark picture of high $T$ regime of QGP, that is, the unbound quark-antiquark propagation in the mesonic channels. For this reason we calculate mesonic correlators using free quark propagators with the same source as for the mesons at $T=2.03 T_{c}$. The behaviour of the meson correlation functions after normalizing them with the corresponding free meson correlators on the lattice is shown in Fig. 3b. We notice that the ratio for pseudo-scalar channel is well above one and rapidly enhanced compared to the corresponding free correlator, which confirms the expectations of the instanton model [15]. For the vector channel the ratio remains close to one, flattens more with increasing $t$ separation and reaches almost a plateau. This behaviour is consistent with the picture of a rapidly melting resonance contribution, which is consistent with our observation at $2.03 T_{c}$ in Fig. 2c. However, for a conclusive signature of such a thermal change at high temperature, calculations in wider range of temperatures are required. We are continuing simulation on larger lattice sizes over a wide range of temperatures to get a better possibility of observing signals for plasma as well as the precise contact with nature.

\section{Acknowledgments}

I would like to express my sincere gratitude to C. DeTar, E. Gregory and S. Gupta for valuable discussions at the Lattice 2010 Conference in Villasimius, Italy. This work is supported in part by the Department of Foreign Academic Affairs of Jinan University. We are grateful for the access to the supercomputing facility at South China University of Technology on 64 nodes of the grid.

\section{References}

[1] C. DeTar, Phys. Rev. D 37, 2328 (1988) 
[2] R. Rapp and J. Wambach, Adv. Nucl. Phys. 25, 1 (2000)

[3] CP-PACS Collaboration, A. Khan, et al., Phys. Rev. D 65, 054505 (2002)

[4] HS Collaboration, H. Lin, et al., Phys. Rev. D 79, 034502 (2009)

[5] BGR Collaboration, G. Engel, et al., arXiv:hep-lat/1005.1748

[6] Ph. de Forcrand et al., Phys. Rev. D 63 (054501) 2001 and references therein

[7] P. Petreczky, J. Phys. G30, 431 (2004)

[8] V. Bornyakov et al., Phys. Rev. D 71, 114504(2005)

[9] Y. Namekawa and Y. Kikukawa, PoS (lattice07) 212, (2007)

[10] T. Umenda, K. Nomura, and H. Matsufuru, Eur. Phys. J. C39S1, 9 (2005)

[11] C. Morningstar and M. Peardon, Phys. Rev. D 56, 4043 (1997)

[12] H. Matsufuru, T. Onogi and T. Umeda, Phys. Rev. D 64, 114503 (2001)

[13] R. Edward and U. Heller, Phys. Lett. B 462, 132 (1999)

[14] CP-PCAS Collaboration, A. Khan et al., Phys. Rev. D 63, 034502 (2001)

[15] T. Schäfer and E. Shuryak, Phys. Rev. D 54, 1099 (1996) 